Background and aims Hypoxic-ischaemic (H-I) brain injury in the human perinatal period often leads to significant long-term neurobehavioral dysfunction in the cognitive and sensory-motor domains. The aim of the present study investigated that effect neuroprotective of different dosages of pentoxifylline in neonatal rat model of HIE.

Methods H/I was performed according to the Levine-Rice model on postnatal seven-day-old. Wistar rat pups were randomly divided into four groups as: sham-operated group $(\mathrm{n}=$ 17), $\mathrm{H} / \mathrm{I}(\mathrm{n}=16), \mathrm{H} / \mathrm{I}$ and intraperitoneal Pentoxifylline $60 \mathrm{mg} /$ kg-treated group $(\mathrm{n}=17)$ and HI and intraperitoneal Pentoxifylline $100 \mathrm{mg} / \mathrm{kg}$-treated group $(\mathrm{n}=17)$. Twenty-three rat pups, twenty-four hours after hypoxia, the animals were killed for histopathological evaluation to detect apoptosis by caspase- 3 immunohistochemistry method. The other rat pups were grown to 11 weeks. The synaptic plasticity and cognitive function of rats were evaluated using long term potentiation (LTP) and Morris water maze (MWM) test on D77-D82, respectively.

Results Pentoxifylline $60 \mathrm{mg} / \mathrm{kg}$ two doses treatment decreased the number of caspase- 3 positive cells that showed the typical morphological features of apoptosis in only hippocampus (p0.05) but, total numbers of degenerative cell significantly diminished.

Conclusions Low dose pentoxifylline treatment is protective against both brain injury and memory impairment and synaptic plasticity.

\section{P0-0401 IL-6 POLYMORPHISM AT POSITION-174 IN NEWBORN INFANTS WITH PERINATAL ARTERIAL ISCHAEMIC STROKE: ASSOCIATION WITH ADVERSE OUTCOME}

${ }^{1} \mathrm{JC}$ Harteman, ${ }^{2} \mathrm{HLDM}$ Willemen, ${ }^{2} \mathrm{CJ}$ Heynen, ${ }^{3} \mathrm{~A}$ Huisman, ${ }^{4} \mathrm{~L}$ Bont, ${ }^{1} \mathrm{~F}$ van Bel, ${ }^{1} \mathrm{MJNL}$ Benders, 'LS de Vries, ${ }^{1} \mathrm{~F}$ Groenendaal. ${ }^{1}$ Neonatology, Wilhelmina Children's Hospital, Utrecht, Netherlands; ' ${ }^{2}$ Laboratory of Neuroimmunology and Developmental Origins of Disease, University Medical Center Utrecht, Utrecht, Netherlands; ${ }^{3}$ Clinical Chemistry and Haematology, University Medical Center Utrecht, Utrecht, Netherlands; ${ }^{4}$ Paediatric Infectious Disease, Wilhelmina Children's Hospital, Utrecht, Netherlands

\subsection{6/archdischild-2014-307384.1047}

Background Inflammation has been proposed as a hallmark in the pathophysiology of stroke. A functional polymorphism in the interleukin (IL)-6 gene at position-174, encoding for the pro-inflammatory cytokine IL-6, is associated with an increased risk of neonatal brain injury or development of cerebral palsy. The aim was to study whether the IL-6-174 G/C polymorphism increased the risk of perinatal arterial ischaemic stroke (PAIS) or subsequent adverse sequelae.

Methods Infants born at or above 37 weeks gestation with PAIS diagnosed by neonatal MRI ( $n=63$ ) were included. Genotyping of the IL-6-174 G/C polymorphism was performed and compared to 1008 random population controls. Perinatal variables of case infants were reviewed.

Results There were no differences in IL-6-174 genotype between infants with PAIS and population controls. In a multivariable analysis, independent risk factors for adverse outcome after PAIS in a middle cerebral artery territory included CG genotype (OR 5.9; 95\% CI 1.02-33.9) and male sex (OR 4.2; 95\% CI 1.04-17.2).

Conclusion The distribution of the IL-6-174 C > $>$ promotor polymorphism did not differ between infants with PAIS and population controls and therefore do not seem to play a role in stroke risk. However, the IL-6-174 GC genotype was more common among infants who had an adverse outcome following PAIS in the middle cerebral artery territory, suggesting that the level of inflammation does play a role in outcome after PAIS. This may be relevant for neuroprotective strategies.

\section{P0-0402 CHALLENGING CURRENT CONCEPTS REGARDING LATERALITY AND DIRECTION OF BOLD SIGNAL CHANGES IN NEONATAL FUNCTIONAL BRAIN IMAGING}

${ }^{1}$ A Heep, ${ }^{2} \mathrm{H}$ Boecker, ${ }^{2} \mathrm{M}$ Born, ${ }^{2} \mathrm{~L}$ Scheef. ${ }^{1}$ Neonatal Neuroscience Group, University of Bristol, Bristol, UK; ${ }^{2}$ Department of Radiology, University of Bonn, Bonn, Germany

\subsection{6/archdischild-2014-307384.1048}

Background Functional magnetic resonance imaging (fMRI) in preterm infants has been introduced as a non-invasive method to study information processing in the developing brain and to identify early signs of dysfunction. Studying the sensorimotor system, fMRI has so far delivered inconsistent brain activation patterns regarding laterality and direction of BOLD signal changes.

Aims To study evoked responses to unilateral passive sensorimotor stimulation in preterm infants using a customised neonatal head coil.

Patients/methods 14 preterm infants born less 30 weeks gestation were scanned using a block design $(10 \times 30 \mathrm{sec}$ ON-OFF $)$ at $3 \mathrm{~T}$ (Achieva, Philips, Best, NL) using a customised neonatal head coil (J. Nordmeyer-Massner and K. Pruessmann, ETH Zürich) at term equivalent age. Data were pre-processed (slice-timecorrection, motion correction, anatomical co-registration with high-resolution T2-weigthed structural images, $6 \mathrm{~mm}^{3}$ smoothing) and analysed on an individual level using SPM8. Only activation clusters surviving $\mathrm{p}<0.05$ (FWE-corrected) were considered as statistically significant.

Results $5 / 14$ scans had to be withdrawn due to movement artefacts $(n=4)$ or technical failure $(n=1)$. In the remaining sample of 9/14 subjects, unilateral passive sensorimotor stimulation elicited primarily positive BOLD responses, which were located in contralateral (8/9) and ipsilateral (1/9) primary sensori-motor cortex (SMC).

Conclusions The study results indicate that improved sensitivity of a size-optimised neonatal head coil is crucial for detection of primary SMC activity on an individual level and question the hypothesis of unfocused negative and bilateral BOLD responses in the premature brain.

\section{PO-0403 USEFULNESS OF THE EVALUATION OF CEREBRAL BLOOD VOLUME TO PREDICT ADVERSE OUTCOMES IN INFANTS WITH ASPHYXIA}

${ }^{1}$ A Hirose, ${ }^{1} \mathrm{~A}$ Kondo, ${ }^{2} \mathrm{~T}$ Takami, ${ }^{1} \mathrm{D}$ Sunohara, ${ }^{1} \mathrm{H}$ Kawashima. ${ }^{1}$ Pediatrics, Tokyo Medical University, Tokyo, Japan; ${ }^{2}$ Neonatology, Toho University, Tokyo, Japan

\subsection{6/archdischild-2014-307384.1049}

Background and aims Increased cerebral haemoglobin oxygen saturation $\left(\mathrm{rSO}_{2}\right)$ and decreased cerebral fractional tissue oxygen 